\title{
Antiplatelet Therapy, Diabetic Neuropathy and Peripheral Vascular Disease: A Unitary Approach
}

Takahisa Deguchi ${ }^{1}$, Raymond L. Rosales ${ }^{1,2 *}$, Teruto Hashiguchi ${ }^{3}$ and Kimiyoshi Arimura ${ }^{1,4}$

${ }^{1}$ Department of Neurology and Geriatrics, Kagoshima University Graduate School of Medical and Dental Sciences, Kagoshima, Japan ${ }^{2}$ Department of Neurology and Psychiatry, University of Santo Tomas, Manila, Philippines

${ }^{3}$ Department of Laboratory and Vascular Medicine, Kagoshima University Graduate School of Medical and Dental Sciences, Kagoshima, Japan

${ }^{4}$ Ohkatsu Neurology and Rehabilitation Hospital, Kagoshima, Japan

\begin{abstract}
Although pathologically not necessarily the same, germane to both diabetic neuropathy and peripheral vascular disease is the morbid vascular complication of diabetes mellitus. As it appears, strict glycemic control alone is unable to neither prevent nor promote recovery of diabetic microvascular complications, thus the need to seek other treatment strategies. In addressing the vascular complications, a unitary approach between diabetic neuropathy, peripheral vascular disease and antiplatelet therapy is in order. Notably, the fine balance between platelet/coagulation and fibrinolysis systems are dominantly shifted to acceleration of the platelet/coagulation system in diabetes mellitus. Not only platelet homophilic aggregation, but also platelet heterophilic aggregation with neutrophils or monocytes in inflammatory conditions is observed. Hyperglycemia also induces overproduction of reactive oxygen species by multiple pathomechanisms that injure endothelial cells and reduce nitric oxide which eventually reduce the blood flow in microvessels. Among other antiplatelet agents, cilostazol is a novel antiplatelet agent that has been found to have beneficial effects in macrovascular events like stroke and peripheral vascular diseases. In peripheral vascular disease clinical trials, there is robust data that cilostazol is efficacious in improving walking speed. Also, cilostazol increases nerve blood flow/nerve conduction, and inhibits reduction in pericyte area of endoneurial microvessels in animal models of diabetic neuropathy. Clinical trials on diabetic polyneuropathy alone or in combination with peripheral vascular disease indicate clinical improvement in blood flow, but not necessarily in neuropathy parameters. Since a neurodegenerative process is equally as important a pathomechanism, the effect of antiplatelet therapy in diabetic neuropathy should be examined in long-term clinical trials that may potentially unfold its benefits over time.
\end{abstract}

Keywords: Antiplatelet therapy; Diabetic neuropathy; Peripheral vascular disease; Diabetic foot; Cilostazol

\section{Introduction}

Microvascular complications characterized by retinopathy, nephropathy and neuropathy are well recognized in both type1 and type2 diabetes. Diabetic microvascular complications account for a significant portion of morbidity and mortality. In the past decade, intensive glycemic control and blood pressure control have shown to significantly reduce the microvascular related morbidity, however, this matter of prevention has not been borne out from large, long term clinical trials $[1,2]$.

Whether stricter glycemic control alone is sufficient to prevent vascular complications in type 2 diabetes is a matter of debate. In one clinical study [3], the intensive glucose lowering arm of the Action to Control and Cardiovascular Risk (ACCORD) trial in type 2 diabetes had been terminated because of increased mortality. In the said study [3] the targeted A1C level was below 6\% and was combined with treatments to increase HDL cholesterol, lower LDL cholesterol and lower blood pressure. Another intensive glycemic control trial (Veteran Affairs Cooperative Study on Glycemic Control and Complications in Type II Diabetes Mellitus; VA-CSDM) [4] showed a non-significant increase in the risk of cardiovascular events. Furthermore, a long-term clinical trial for 5.6 years with intensive but relatively milder (targeted A1C 6.9\%) glycemic control in type 2 diabetes (VADT) showed no significant effect on the rates of major cardiovascular events, death or microvascular complications, with the exception of progression of albuminuria [5]. These results suggest that strict glycemic control alone could not prevent or nor recover patients from diabetic microvascular complications, but may increase mortality. Stirban [6] suggested that: 1) lowering blood glucose in elderly and pluri-morbid patients should be performed with caution, 2) combination therapies should be rigorously considered, and 3) there may be a certain threshold beyond which lowering of blood glucose may be detrimental.

Along these conclusions, additive treatments with appropriate glycemic control will be needed for microvascular complications other than blood pressure control. In a multifactorial intervention study in type 2 diabetes for 13.3 years, intensive intervention with multiple drug regimens (antihypertensive drugs, statin, fibrate and aspirin) and behavior modification had sustained beneficial effects with respect to vascular complications, including microvasculopathy and on rates of death from any cause and from cardiovascular causes [7].

This present review highlights a unitary approach between the vascular complications such as diabetic neuropathy and peripheral arterial vascular disease (PAD), with the possible positive effects of antiplatelet therapy.

\section{Platelet and Coagulation/Fibrinolysis Systems in Diabetes}

Platelets participate in the blood haemostasis in case of blood

${ }^{*}$ Corresponding author: Raymond L. Rosales, MD, PhD, Department of Neurology and Psychiatry, Faculty of Medicine and Surgery, University of Santo Tomas, Manila, Philippines, 1008, E-mail: rrrosales@mnl.ust.edu.ph

Received November 30, 2011; Accepted January 17, 2012; Published January 22, 2012

Citation: Deguchi T, Rosales RL, Hashiguchi T, Arimura K (2012) Antiplatelet Therapy, Diabetic Neuropathy and Peripheral Vascular Disease: A Unitary Approach. J Diabetes Metab S5:005. doi:10.4172/2155-6156.S5-005

Copyright: ( 2012 Deguchi T, et al. This is an open-access article distributed unde the terms of the Creative Commons Attribution License, which permits unrestricted use, distribution, and reproduction in any medium, provided the original author and source are credited. 
vessel injury accompanied by coagulation factors. The fibrinolysis system regulates the thrombus formation on endothelial cells resulting in maintenance of vascular homeostasis. The important concept of applying the platelet/coagulation system as well as the fibrinolysis system to vascular biology is that these fine systems always work even in healthy conditions, where endothelial cells are always damaged by shear stress under the blood flow and are always needed to be repaired by the same systems. Interestingly, this fine balance between the platelet/coagulation and the fibrinolysis systems are dominantly shifted towards the platelet/coagulation system acceleration induced by, among others, aging, hypertension, obesity, and especially by diabetic states. In addition to haemostatic factors, platelets contain several kinds of growth factors, such as vascular endothelial growth factor-A (VEGF-A), platelet derived growth factor (PDGF) and transforming growth factor-beta (TGF- beta). All of these growth factors have roles in vascular repair and maintenance. Platelet VEGF-A is a major component of serum VEGF-A [8] and serum VEGF-A significantly increases in patients with diabetic neuropathy during the neurologically active symptomatic stages [9]. These observations suggest the role of platelet VEGF-A on neuron homeostasis in diabetic neuropathy [9]. Pattern recognition receptors (RAGE [Receptor for Advanced Glycation Endproducts]) are known to be key molecules in a recent concept called "glucose memory" [10]. Hyperglycemia per se induces increased generation of advanced glycation end products (AGEs). The ligands for RAGE include AGEs, HMGB1 (high mobility group protein B1), and amyloid- beta -protein. RAGE is expressed on endothelial cells and its intra-cellular signaling leads to NF-kappaB activation, indicative of pro-inflammatory endothelial cell activation. From the recently reported RAGE expression on platelet membranes and since platelets are activated by AGEs through RAGE [10], a pathological link has been established between hyperglycemia, AGE, "glucose memory" and platelet activation. In effect, all these cascade of events may lead to a disturbance in microvascular circulation of patients with diabetes (Figure 1).

\section{Platelet heterophilic aggregation in diabetes}

From another aspect of platelet haemostatic function, a new concept of platelet biology has recently been developed. Not only platelet homophilic aggregation, but also platelet heterophilic aggregation with neutrophils or monocytes in inflammatory conditions are observed, and these heterophilic aggregations are closely associated with the innate immune system [11]. Ligand-receptor binding between Gramnegative bacterial Lipopolysaccharide (LPS) and Toll like receptor 4 expressed on platelet membrane activate platelets and form plateletneutrophil aggregation [11]. On the other hand, platelet-monocyte aggregation by P-selectin/ PSGL-1 (P-Selectin Glycoprotein Ligand 1) binding leads to the stabilization of COX-2 mRNA in monocytes and prolongs the inflammatory milieu [12]. Hyperglycemia reportedly also induce both platelet-neutrophil and platelet-monocyte aggregation [13], and that AGEs reportedly induce platelet-neutrophil aggregation [14]. Although further studies may be required on the subject of platelet heterophilic aggregations in diabetic conditions, this new platelet biology of heterophilic binding to another type of cells suggest a close contribution to diabetic vascular complications, especially in understanding microvasculopathy.

\section{De novo synthesis of platelet tissue factor in diabetes}

Since platelets do not have a nucleus, platelets have been thought to have no capacity of protein de novo synthesis. Contrary to this previous concept, tissue factor (TF), which is an initiation factor of the extrinsic coagulation cascade, is produced in activated platelets through TF pre-mRNA splicing [15]. Gerrits and colleagues [16] reported that TF synthesis in platelets is inhibited by insulin in healthy individuals, but in patients with type 2 diabetes, inhibition is impaired. An increased amount of TF expression on platelets in patients with type 2 diabetes than in healthy individuals should be one of the mechanisms for macroand microvascular complications of diabetes.

\section{Platelet micro-particles in diabetes}

Micro-particles derived from several cell lines including platelets and endothelial cells provide a new hypothesis for the pro-coagulant state in diabetic patients. Micro-particles are membrane fragments shed from damaged or activated cells. Platelet micro-particles (PMPs) bearing rich amount of TF in diabetic patients are strongly suggested to

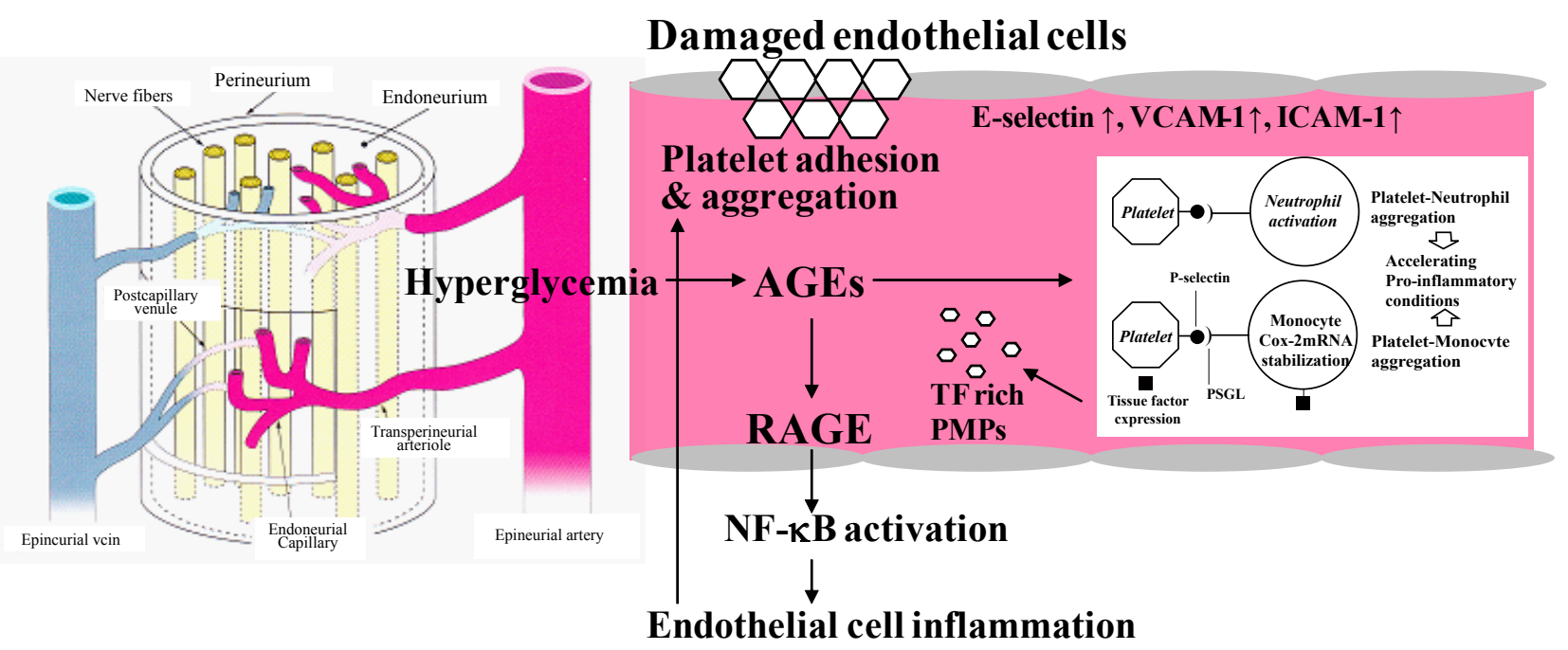

Figure 1: The effects of hyperglycemia in the peripheral nerve, vascular supply and platelet function; AGEs: advanced glycation endproducts; PMPs: platelet microparticles; RAGE: Receptor for Advanced Glycation Endproducts; TF: tissue factor. 
promote the progression of macro- and microangiopathy [17].

\section{Diabetic Neuropathy, Pathophysiology, and Antiplatelet Therapy}

\section{Risk factors and glycemic control in diabetic neuropathy}

Vascular complications in diabetic neuropathy range from focal neuropathies (e.g. cranial nerves, femoral nerves) to symmetric polyneuropathies (DPN or diabetic polyneuropathy). All of the microvascular complications occurring in neuropathies (including autonomic neuropathy) in diabetic patients are categorically strong risk factors in the multivariable models, exceeding the effect of traditional risk factors [18]. In fact, previous studies indicated that peripheral neuropathy was related to mortality in diabetes $[19,20]$. These findings implicate that treatment of diabetic neuropathy as early as possible is highly recommended to prevent early mortality. The main strategy of the preventing neuropathy is believed to introduce a strict glycemic control. The strict glycemic control can decrease the incidence of macrovasculopathy and microvasculopathy, including retinopathy and nephropathy, but not necessarily neuropathy. The implication of these findings is that additive treatments other than glycemic control may be needed. Furthermore, intensive intervention with multiple drug regimens also could not entirely prevent nor allow recovery from neuropathy [7]. Thus, it is imperative that new treatment strategies ought to be developed in diabetic neuropathy.

\section{Pathophysiology of diabetic neuropathy}

The prevalence of diabetic neuropathy may approach approximately $50 \%$ in patients with longer-duration of illness. Chronic, symmetric sensori-motor polyneuropathy (DPN) and autonomic neuropathy are the most common forms of diabetic neuropathy. In the pathophysiology of DPN, direct metabolic dysfunction of cell and axon, as well as microvasculopathy, have been explored. Axonopathy are present in both myelinated and unmyelinated fibers, with early development of Schwann cell-axon networks, and later distal axonopathy. In tandem, alternations in microvessels are observed in peripheral nerves. Reduced blood flow due to autonomic nerve dysfunction may also contribute to the on-going neuropathic process [21]. It is obvious then that the pathophysiology of DPN is multi-factorial. The metabolic effects of chronic hyperglycemia and the consequences of ischemia on the peripheral nerve are likely to be critical factors that lead to neuro-axonal dysfunction and damage. Also, a wide range of pathomechanisms in DPN have been put forward that include activation of polyol pathway and protein kinase $\mathrm{C}$ (PKC), generation of reactive oxygen species (ROS) (oxidative stress), reactive nitrogen species, and AGEs [22]. Microvasculopathy in peripheral nerves are also multifactorial. Oxidative stress has been considered the final common pathway of cellular injuries in hyperglycemia. Several mechanisms of hyperglycemia-induced cellular injury were described in the vascular endothelium [23]. Hyperglycemia induces overproduction of ROS by the mitochondrial electron transport chain. Superoxides bind to nitric oxide (NO) that produce the strong oxidant peroxynitrite and injure endothelial cells. The reduced availability of endothelial NO causes the prevention of vasodilation which antagonizes thrombosis and antiinflammatory properties. NO also inhibits the production of the potent vasodilator peptide endothelin-1.

Hyperglycemia is known to stimulate formation of diacylglycerol, which leads to the activation of PKC [24]. The oxidizing environment and increased carbohydrate accumulation in diabetes accumulate AGEs with the reduction of clearance from plasma [21]. The basement membrane of endothelial cells becomes glycosylated thereby contributing to impaired vasodilatation. Furthermore, AGEs bind to cell surface receptor for AGE (RAGE) and initiate an inflammatory signaling pathway that further increases oxidative stress and injures both neuronal and endothelial cells. These findings results in an imbalance of vasoactive agents and impair microvascular perfusion in nerves. A prospective study indicates that, apart from glycemic control, the incidence of neuropathy is associated with potentially modifiable cardiovascular risk factors, including a raised triglyceride level, bodymass index, smoking, and hypertension [25]. Linked to the vascular pathophysiologic mechanism of DPN, many treatment options have been proposed including a PKC-beta inhibitor, reduction of AGEs, VEGF and angiotensin converting enzyme inhibitors [22]

\section{Antiplatelet therapy with cilostazol in diabetic neuropathy}

A parallel involvement of both nerves and blood vessels may be the rule than the exception in diabetic neuropathy [26,27]. In the light of the foregoing issues of platelet involvement, antiplatelet therapy would figure as a likely candidate to improve the microvascular milieu in DPN. The 2-oxo-quinolone derivative cilostazol (Pletaal ${ }^{\circ}$ ) is a selective and reversible inhibitor of phophodiesterase $3 \mathrm{~A}$, with both antiplatelet and vasodilatory properties [28]. Cilostazol is a novel anti-platelet agent that has been found to have beneficial effects in macrovascular events like stroke and PAD.

Hinged on the vascular physiologic mechanism of DPN, a number of experimentally induced diabetes studies in animals demonstrated that cilostazol might have beneficial effects in that the drug inhibits platelet aggregation and leads to vasodilatation [28]. Through an improved nerve Na-K ATPase activity and cyclic adenosine monophosphate (cAMP) content, cilostazol increases nerve blood flow/nerve conduction, and inhibits reduction in pericyte area of endoneurial microvessels in animal models [29-35]. For instance, in a dose- and time-dependant manner, separate administrations of both locally applied and dietary cilostazol increased nerve blood flow [29], improved nerve conductions by restoring nerve $\mathrm{Na}-\mathrm{K}$ ATPase activity $[30,32,33]$, and allowed axonal regeneration [35]. While there are several published clinical trials (with reviews) on the benefits of cilostazol for PAD, such is not the case for the same drug in DPN [36]. Few published cilostazol studies in human participants with DPN are largely constrained by lack of randomization and comparator/placebo arms, selection bias, limited study population and lack of robust outcome parameters [33,37-39]. In addressing these main study issues, cilostazol was systematically studied in the most recent randomized placebo-controlled trial (ASCEND: Asian Study on Cilostazol Safety and Clinical Efficacy in Neuropathies of Diabetes Mellitus Type 2) [40], in which the primary efficacy parameter was a change in neuropathy symptom scores and the secondary efficacy parameter was a change in walking speed from baseline to week 12 . The safety parameters were changes in nerve conduction studies as well as reporting of adverse events. The results indicate that despite a significant improvement in the neuropathy symptom scores in the overall motor and sensory categories of the 3 arms of the study (placebo, cilostazol low dose $100 \mathrm{mg} /$ day and high dose $200 \mathrm{mg} /$ day) from baseline to week 12 , no significant differences were found among the groups. In this pilot Asian study, there is thus non-superiority of cilostazol in regard to improvement of neuropathy symptoms over placebo in the short study span. Interestingly, cilostazol, at low dose, was effective in improving walking speed from baseline to week 12, implying an improved blood flow. No significant worsening nor improvement in motor and sensory nerve conduction parameters were observed, Comparing the 3 study arms from baseline to weeks 4,12 , and 16 , no significant worsening nor 
improvement in motor and sensory nerve conduction parameters were observed, supporting cilostazol's safety. From the ASCEND study, one may be able to derive the following insights: (1) Cilostazol, with tight glycemic control, may have the added benefit of improving blood flow; (2) It may take time and a longer observation study period to possibly see effects of cilostazol in DPN, considering the trends (but not to significant levels compared to placebo) of improvement in motor and sensory neuropathy scores, and (3) Autonomic manifestations in DPN may have an independent pathophysiology, considering that it did not show improvement in the entire study period.

Another prospective double-blinded, randomized, placebocontrolled clinical trial assessed the effects of cilostazol on neuropathic symptomatology in patients with coexisting and established PAD and DPN [36]. In this perhaps the longest prospective study to date, no significant difference was found between the placebo and cilostazol groups, using the Toronto Clinical Neuropathy Scoring System and Vibration Perception Thresholds at 6 and 24 weeks. The common denominator between the ASCEND trial [40] and the aforementioned PAD and DPN trial [36] is the tight glycemic control and medically optimized cohort of patients enrolled in both studies.

\section{Peripheral Artery Disease and Antiplatelet Therapy \\ Diabetic foot}

Diabetic foot is an intractable complication of diabetes mellitus due to decrease in blood flow of lower limbs. Occurrence of diabetic foot is multifactorial, that includes presence of autonomic neuropathy, arteriosclerosis and concomitant infection due to reduced resistance to bacteria with hyperglycemia. If there is diabetes, the relative risk of PAD is 2-4 times higher than healthy subjects [25]. In diabetes, other independent risk factors for PAD including age, increased systolic blood pressure, reduced HDL cholesterol, smoking, prior cardiovascular disease, peripheral sensory neuropathy, and retinopathy, were also reported. Each $1 \%$ increase in A1c was associated with a $28 \%$ increased risk of PAD [41]. However, normoglycemic neuropathy subjects have significantly more features of metabolic syndrome (other than hyperglycemia) than diabetics [42]. Thus, it appears that neuropathy, one of the microvasculopathy in diabetes, is an important factor for PAD.

\section{Endothelial dysfunction and PAD}

Endothelial dysfunction due to hyperglycemia or insulin resistance may lead to the development of neuropathy [43]. Hyperglycemia induces a pro-coagulant effect irrespective of insulin levels, whereas hyperinsulinemia inhibits fibrinolysis irrespective of glucose levels [44]. These findings suggest that patients with hyperglycemia due to insulin resistance are especially susceptible to thrombotic events by a concurrent insulin-driven impairment of fibrinolysis and a glucosedriven activation of coagulation. In diabetes, the overlapping features of impaired vascular endothelium, platelet activation, increased activity of coagulation factors, changes in the structure of fibrin and decreased fibrinolytic activity, lead to a vicious cycle that further impair platelet function [45]. Thus, in the case of diabetic foot, glycemic control and vasodilator drugs (e.g. antiplatelet drugs, prostaglandin drugs) that improve blood flow, may be the key therapeutic approaches. While drugs which improve blood flow in diabetic neuropathy may be in the forefront, other approaches such as, gene therapy and cell therapy that promote angiogenesis may be another way in the future [46,47].

\section{Antiplatelet drugs for PAD}

According to both the Inter-Society Consensus for the Management of Peripheral Arterial Disease (TASC II) and the American Cardiology and Heart Associations (ACC/AHA) guidelines, antiplatelet drugs should be administered for all patients with symptomatic PAD in order to reduce the risk of cardiovascular morbidity and mortality, (Grade A) [48]. Antiplatelet drug therapy for patients with PAD can be expected to be also effective prophylaxis of cardiovascular disease. Thus antiplatelet drugs will be used to manage all patients with PAD. Overall, among high risk patients, an allocation to antiplatelet therapy potentially reduce the combined outcomes of any serious vascular events and vascular mortality.

Aspirin: Low dose aspirin (75-150 mg daily) is an effective antiplatelet regimen for long term use in occlusive vascular events, but in acute settings, an initial loading dose of at least $150 \mathrm{mg}$ aspirin may be required [49]. On the other hand, among 3350 participants without clinical cardiovascular disease, identified with a low anklebrachial index (based on screening a general population-The Aspirin for Asymptomatic Atherosclerosis trial), the administration of aspirin compared with placebo did not result in a significant reduction in vascular events [50]. However, All asymptomatic patients and symptomatic patients with evidences of atherosclerosis in other circulatory beds should be prescribed an antiplatelet drug (Grade A) [25]. Asymptomatic patients without evidence for atherosclerotic disease elsewhere may be considered for antiplatelet therapy (Grade C) [25]. The ACC/ AHA guidelines recommend the administration of aspirin on individuals with or without clinical signs of other cardiovascular disease (Grade A) [51].

Clopidogrel: Clopidogrel is an inhibitor of platelet $\mathrm{P} 2 \mathrm{Y} 12$ receptor similar to ticlopidine, but is considered a safer drug. In a randomized, blinded, trial of clopidogrel versus aspirin in patients at risk of ischemic events (CAPRIE), long-term administration of clopidogrel to patients with atherosclerotic vascular disease is more effective than aspirin in reducing the combined risk of ischemic stroke, myocardial infarction, or vascular death, and the overall safety profile of clopidogrel is at least as good as that of medium-dose aspirin [52]. Nevertheless, aspirin is generally considered the antiplatelet drug of choice because of the high incidence of coronary disease and its lower cost. As antiplatelet therapy like cilostazol, both aspirin and clopidogrel conceivably may be options for PAD, and perhaps even DPN. Apart from the fact that both lack vasodilatory effects, and if one were to pursue platelet aggregation methodological studies, occurrence of aspirin and clopidogrel resistance may reduce their respective efficacies [53]

Novel inhibitor of platelet P2Y12 receptor, prasugrel and ticagrelor are reported to have favorable effects after percutaneous coronary intervention compared with clopidgrel [54]. However, we await to this time whether these drugs will also achieve the desired effect in PAD, even if both drugs may have shown a stronger protection, efficacy and safety among diabetic patients.

Cilostazol: Cilostazol, a phosphodiesterase inhibitor, suppresses platelet aggregation and also acts as a direct arterial vasodilator. Cilostazol improved walking distances, significantly increasing initial claudication distance and absolute claudication distance [55,56]. Compared with placebo, long-term use of cilostazol, $100 \mathrm{mg}$ or $50 \mathrm{mg}$ twice a day, significantly improves walking distances in patients with intermittent claudication [57]. Based upon the evidence of benefit, a therapeutic trial of cilostazol is recommended to improve symptoms and increase walking distance in patients with claudication, particularly if previous antiplatelet agents were ineffective $[25,51,58]$. 


\section{Conclusion}

There leaves no doubt that platelet function and regulation do have important roles in diabetes mellitus per se, DPN and PAD. The jury has arrived as regard efficacy of cilostazol, more than other antiplatelet agents in the realm of PAD. However, in the realm of DPN, much work has yet to be established to reach robustness of efficacy. The situation changes with patients having coexistent PAD and DPN, both at different stages together. An improved blood flow with antiplatelet therapy may either be delayed or not be reflected in nerve blood flow and thus not transmute to alleviation of neuropathy symptoms. Moreover, one should be aware that in DPN in particular, not only vascular but neurodegenerative mechanisms (whether in association with metabolic disturbances or not) come into play, such that there may be an inconsistent or perhaps delayed response to medications.

\section{Acknowledgement}

Michelle Joya-Tanglao, PTRP kindly assisted in the literature search as well as manuscript preparation. The CNS staff of Metropolitan Medical Center (Manila) assisted RLR in many ways to complete this work.

\section{References}

1. Nathan DM, Cleary PA, Backlund JY, Genuth SM, Lachin JM, et al. (2005) Intensive diabetes treatment and cardiovascular disease in patients with type 1 diabetes. N Engl J Med 353: 2643-2653.

2. UK Prospective Diabetes Study (UKPDS) Group (1998) Intensive bloodglucose control with sulphonylureas or insulin compared with conventional treatment and risk of complications in patients with type 2 diabetes (UKPDS 33). Lancet 352: 837-853.

3. Mayor S (2008) Intensive glucose lowering arm of diabetes trial is stopped after excess deaths. BMJ 336: 407.

4. Abraira C, Colwell J, Nuttall F, Sawin CT, Henderson W, et al. (1997) Cardiovascular events and correlates in the Veterans Affairs Diabetes Feasibility Trial. Veterans Affairs Cooperative Study on Glycemic Control and Complications in Type II Diabetes. Arch Intern Med 157: 181-188.

5. Duckworth W, Abraira C, Moritz T, Reda D, Emanuele N, et al.(2009) Glucose control and vascular complications in veterans with type 2 diabetes. $\mathrm{N}$ Engl J Med 360: 129-139.

6. Stirban A, Tschoepe D, Stratmann B (2009) Shifting the disease management paradigm from glucose: what are the pros? Diabetes Care 32: S349-S352.

7. Gaede P, Lund-Andersen H, Parving HH, Pedersen $\mathrm{O}$ (2008) Effect of a multifactorial intervention on mortality in type 2 diabetes. N Engl J Med 358: 580-591.

8. Kimura K, Hashiguchi T, Deguchi T, Horinouchi S, Uto T, et al. (2007) Serum VEGF - as a prognostic factor of atherosclerosis. Atherosclerosis 194: 182-188.

9. Deguchi T, Hashiguchi T, Horinouchi S, Uto T, Oku H, et al. (2009) Serum VEGF increases in diabetic polyneuropathy, particularly in the neurologically active symptomatic stage. Diabet Med 26: 247-252.

10. Yamagishi S, Matsui T (2010) Smooth muscle cell pathophysiology and advanced glycation end products (AGEs). Curr Drug Targets 11: 875-881.

11. Clark SR, Ma AC, Tavener SA, McDonald B, Goodarzi Z, et al. (2007) Platelet TLR4 activates neutrophil extracellular traps to ensnare bacteria in septic blood. Nat Med 13: 463-469.

12. Dixon DA, Tolley ND, Bemis-Standoli K, Martinez ML, Weyrich AS, et al. (2006) Expression of COX-2 in platelet-monocyte interactions occurs via combinatorial regulation involving adhesion and cytokine signaling. J Clin Invest 116: $2727-$ 2738.

13. Vaidyula, VR, Boden G, Rao AK (2006) Platelet and monocyte activation by hyperglycemia and hyperinsulinemia in healthy subjects. Platelets 17: 577-585.

14. Gawlowski T, Stratmann B, Stirban AO, Negrean M, Tschoepe D (2007) AGEs and methylglyoxal induce apoptosis and expression of Mac-1 on neutrophils resulting in platelet-neutrophil aggregation. Thromb Res 121: 117-126.

15. Schwertz H, Tolley ND, Foulks JM, Denis MM, Risenmay BW, et al. (2006) Signal-dependent splicing of tissue factor pre-mRNA modulates the thrombogenicity of human platelets. J Exp Med 203: 2433-2440.
16. Gerrits AJ, Koekman CA, van Haeften TW, Akkerman JW (2010) Platelet tissue factor synthesis in type 2 diabetic patients is resistant to inhibition by insulin. Diabetes 59: 1487-1495.

17. Csongradi E, Nagy BJ, Fulop T, Varga Z, Karanyi Z, et al. (2011) Increased levels of platelet activation markers are positively associated with carotid wall thickness and other atherosclerotic risk factors in obese patients. Thromb Haemost 106: 683-692.

18. Soedamah-Muthu SS, Chaturvedi N, Witte DR, Stevens LK, Porta M, et al (2008) Relationship between risk factors and mortality in type 1 diabetic patients in Europe: the EURODIAB Prospective Complications Study (PCS) Diabetes Care 31: 1360-1366.

19. Coppini DV, Bowtell PA, Weng C, Young PJ, Sönksen PH (2000) Showing neuropathy is related to increased mortality in diabetic patients- a survival analysis using an accelerated failure time model. J Clin Epidemiol 53: 519-523.

20. Forsblom CM, Sane T, Groop PH, Tötterman KJ, Kallio M, et al. (1998) Risk factors for mortality in Type II (non-insulin-dependent) diabetes: evidence of a role for neuropathy and a protective effect of HLA-DR4. Diabetologia 41: 1253-1262.

21. Shakher J, Stevens MJ (2011) Update on the management of diabetic polyneuropathies. Diabetes Metab Syndr Obes 4: 289-305.

22. Brownlee M (2001) Biochemistry and molecular cell biology of diabetic complications. Nature 414: 813-820.

23. Brownlee M (2005) The pathobiology of diabetic complications: a unifying mechanism. Diabetes 54: 1615-1625.

24. Tesfaye S, Chaturvedi N, Eaton SEM , Ward JD, Manes C, et al. (2005) Vascular risk factors and diabetic neuropathy. N Engl J Med 352: 341-350.

25. Norgren L, Hiatt WR, Dormandy JA, Nehler MR, Harris KA, et al. (2007) InterSociety Consensus for the Management of Peripheral Arterial Disease (TASC II). J Vasc Surg 45: S5-S67.

26. Yagihashi S (1995) Pathology and pathogenetic mechanisms of diabetic neuropathy. Diabetes Metab Rev 11: 193-225.

27. Zochodne DW (2007) Diabetes mellitus and the peripheral nervous system manifestations and mechanisms. Muscle Nerve 36:144-166.

28. Schror K (2002) The pharmacology of cilostazol. Diabetes Obes Metab 2 S14-S19.

29. Kihara M, Schmelzer JD, Low PA (1995) Effect of cilostazol on experimenta diabetic neuropathy in the rat. Diabetologia 38: 914-918.

30. Naka K, Sasaki H, Kishi Y, Furuta M, Sanke T, et al. (1995) Effects of cilostazol on development of experimental diabetic neuropathy: functional and structural studies, and Na-KATPase acidity in peripheral nerve in rats with streptozotocininduced diabetes. Diabetes Res Clin Pract 30: 153-162.

31. Sekiguchi M, AokiY, Konno S, Kikuchi S (2008) The effects of cilostazol on nerve conduction velocity and blood flow: acute and chronic cauda equine compression in a canine model. Spine 33: 2605-2611.

32. Suh KS, Oh SJ, Woo JT, Kim SW, Yang IM, et al. (1999) Effect of cilostazol on the neuropathies of streptozotocin-induced diabetic rats. Korean J Intern Med 14: $34-40$

33. Takei I, Nakamoto S, Ishii M, Yamauchi A, Tokui M, et al. (1997) Cilostazol, a phosphodiesterase inhibitor for the treatment of diabetic neuropathy. Diabetes Res 32: 19-25.

34. Uehara K, Sugimoto K, Wada R, Yoshikawa T, Marukawa K, et al. (1997) Effects of cilostazol on the peripheral nerve function and structure in STZinduced diabetic rats. J Diabetes Complications 11: 194-202.

35. Yamamoto $Y$, Yasuda Y, Kimura Y, Komiya $Y$ (1998) Effects of cilostazol, an antiplatelet agent, on axonal regeneration following nerve injury in diabetic rats. Eur J Pharmacol 352: 171-178

36. O'Donnell ME, Badger SA, Sharif MA, Makar RR, Young IS, et al. (2009) The effects of cilostazol on peripheral neuropathy in diabetic patients with peripheral arterial disease. Angiology 59: 695-704.

37. Kimura M (1996) Effects of Pletal on incidence of sensory dysfunctions in diabetics. J New Remedies Clin 45: 371-376. 
Citation: Deguchi T, Rosales RL, Hashiguchi T, Arimura K (2012) Antiplatelet Therapy, Diabetic Neuropathy and Peripheral Vascular Disease: A Unitary Approach. J Diabetes Metab S5:005. doi:10.4172/2155-6156.S5-005

38. Okuda Y, Mizutani M, Ikegami T, Ueno E, Yamashita K (1992) Hemodynamic effects of cilostazol on peripheral artery in patients with diabetic neuropathy. Arzneimittelforschung 42(4): 540-542.

39. Sekiguchi M, Morikawa A, Nakajima K, Ito H (1991) Clinical usefulness of cilostazol (Pletaal) on diabetic neuropathy and serum lipids levels. Jpn Pharmacol Ther 19(5): 3273-3277.

40. Rosales RL, Delgado-Delos Santos MM, Mercado-Asis LB (2011) Cilostazol: A Pilot Study on Safety and Clinical Efficacy in Neuropathies of Diabetes Mellitus Type 2 (ASCEND). Angiology 62: 625-635.

41. Adler Al, Stevens RJ, Neil A, Stratton IM, Boulton AJM, et al. (2002) UKPDS 59: hyperglycemia and other potentially modifiable risk factors for peripheral vascular disease in type 2 diabetes. Diabetes Care 25: 894-899.

42. Smith AG, Rose K, Singleton JR (2008) Idiopathic neuropathy patients are at high risk for metabolic syndrome. J Neurol Sci 273: 25-28.

43. Singleton JR, Smith AG, Russell JW, Feldman EL (2003) Microvascular complications of impaired glucose tolerance. Diabetes 52: 2867-2873.

44. Stegenga ME, van der Crabben SN, Levi M, de Vos AF, Tanck MW, et al. (2006) Hyperglycemia stimulates coagulation, whereas hyperinsulinemia impairs fibrinolysis in healthy humans. Diabetes 55: 1807-1812.

45. Grant PJ (2007) Diabetes mellitus as a prothrombotic condition. J Intern Med 262: $157-172$

46. Schratzberger P, Walter DH, Rittig K, Bahlmann FH, Pola R, et al. (2001) Reversal of experimental diabetic neuropathy by VEGF gene transfer. $\mathrm{J}$ Clin Invest 107: 1083-1092.

47. Naruse K, Hamada Y, Nakashima E, Kato K, Mizubayashi R, et al. (2005) Therapeutic neovascularization using cord blood-derived endothelial progenitor cells for diabetic neuropathy. Diabetes 54: 1823-1828.

48. Mohler III E, Giri J (2008) Management of peripheral arterial disease patients: comparing the ACC/AHA and TASC-II guidelines. Curr Med Res Opin 24: 2509-2522.
49. Antithrombotic Trialists' Collaboration (2002) Collaborative meta-analysis of randomised trials of antiplatelet therapy for prevention of death, myocardial infarction, and stroke in high risk patients. BMJ 324: 71-86.

50. Fowkes FGR, Price JF, Stewart MCW, Butcher I, Leng GC, et al. (2010) Aspirin for prevention of cardiovascular events in a general population screened for a low ankle brachial index: a randomized controlled trial. JAMA 303: 841-848.

51. Hirsch AT, Haskal ZJ, Hertzer NR, Bakal CW, Creager MA, et al. (2006) ACC/AHA guidelines for the management of patients with peripheral arterial disease (lower extremity, renal, mesenteric, and abdominal aortic) J Vasc and Interventional Radiology 17: 1383-1398.

52. CAPRIE Steering Committee (1996) A randomised, blinded, trial of clopidogre versus aspirin in patients at risk of ischaemic events (CAPRIE). Lancet 348: 1329-1339.

53. Uchiyama S (2011) Clopidogrel resistance: identifying and overcoming a barrier to effective antiplatelet treatment. Cardiovasc Ther 29: e100-e111.

54. Bellemain-Appaix A, Brieger D, Beygui F, Silvain J, Pena A, et al (2010) New P2Y12 inhibitors versus clopidogrel in percutaneous coronary intervention: a meta-analysis. J Am Coll Cardiol 56:1542-1551.

55. Dawson DL, Cutler BS, Meissner MH, Strandness DE (1998) Cilostazol has beneficial effects in treatment of intermittent claudication: results from a multicenter, randomized, prospective, double-blind trial. Circulation 98: 678686.

56. Money SR, Herd JA, Isaacsohn JL, Davidson M, Cutler B, et al. (1998) Effect of cilostazol on walking distances in patients with intermittent claudication caused by peripheral vascular disease. J Vasc Surg 27: 267-275.

57. Beebe HG, Dawson DL, Cutler BS, Herd JA, Strandness DEJ, et al. (1999) A new pharmacological treatment for intermittent claudication: results of a randomized, multicenter trial. Arch Intern Med 159: 2041-2050.

58. Sobel M, Verhaeghe R (2008) Antithrombotic therapy for peripheral artery occlusive disease: American College of Chest Physicians Evidence-Based Clinical Practice Guidelines ( $8^{\text {th }}$ edition). Chest 133: 815S-843S. 